\title{
Hábitos y prácticas sobre la lectura y la escritura de los alumnos del plantel 1 “Gabino Barreda” de la Escuela Nacional Preparatoria de la Universidad Nacional Autónoma de México
}

Lic. Alejandro Ramos Trejo, México. https://orcid.org/0000-0002-3822-6975

alejandro.ramos@enp.unam.mx

Mtra. Lourdes Josefina Avendaño Reyes, México.

https://orcid.org/0000-0002-1661-0079

lourdes.avendano@enp.unam.mx

Mtra. Eva Luz Irene Luna Vargas, México.

eva.luna@enp.unam.mx

https://orcid.org/0000-0002-4681-1031

Mtra. Mónica Chaparro Esquivel, México.

https://orcid.org/0000-0002-8451-2286

monica.chaparro@enp.unam.mx

Escuela Nacional Preparatoria Plantel 1 “Gabino Barreda”, Universidad Nacional Autónoma de México

Recibido: 16 de septiembre del 2020

Aceptado: 16 de octubre del 2020

\section{Resumen}

En el mundo actual, la sociedad está inundada de una gran cantidad de textos orales y escritos, además accede a diversos tipos de escritos mediante múltiples dispositivos como los smartphones, las tabletas, computadoras, sin dejar a un lado las publicaciones editadas como libros, periódicos y revistas. Ante este escenario, la escuela enfrenta un reto mayor para enseñar a leer y escribir. En este contexto, la Escuela Nacional Preparatoria (ENP) realizó diversas actividades como parte de su Proyecto de Modificación Curricular entre el 2012 y 2018. Una de esas actividades se implementó en 2016 durante el Seminario de Análisis y Desarrollo de la Enseñanza en el cual los profesores de los diferentes colegios académicos de la ENP abordaron como tema central "La transversalidad en la actualización de los programas de estudio de la ENP" a partir del análisis de los cinco ejes transversales para fortalecer la formación del bachiller: lectura y escritura, comprensión de textos en otra lengua extranjera, habilidades para la investigación, aprendizaje y 
uso de las TIC (Tecnologías de la Información y Comunicación) y el desarrollo de valores. Por su parte, las profesoras y profesores del Colegio de Geografía del plantel 1 "Gabino Barreda" desarrollaron el tema de la lectura y escritura de textos para aprender y pensar en las asignaturas geográficas del plan de estudios vigente. Por lo tanto, en el presente artículo se muestra un panorama conceptual de la importancia de las habilidades de la lecto-escritura en la formación del bachiller y los resultados de una encuesta aplicada a una muestra de alumnas y alumnos del plantel 1 de la ENP en relación con sus hábitos y prácticas de la lectura y la escritura que desarrollan, tanto en Geografía como en otras asignaturas y los cuales denotan retos para fortalecer la lectura y redacción de textos, así como la búsqueda de información.

Palabras clave: Educación Media Superior, Bachillerato, hábitos de lectura y escritura, Enseñanza de la geografía

\section{Reading and writing habits in the students of the Campus 1 "Gabino Barreda" of the Escuela Nacional Preparatoria of the National Autonomous University of Mexico (UNAM)}

\section{Abstract}

In the actual world, society is overwhelmed with information delivered in a variety of formats such as writings and conferences, gaining immediate access to it through smartphones, tablets, computers and even still in books, newspapers and journals. Given this situation, schools and educational centers face a greater challenge to teach how to read and write. In this context, the Escuela Nacional Preparatoria (ENP) performed different activities as part of the Curriculum Modification Proyect in 2012-2018. Furthermore, in 2016, during the Analysis and Teaching Development Seminar, the teachers of the different educational centers of the ENP addressed as a central topic "The transversality when updating educational programs of the ENP" based on the analysis of the 5 transversal axis to strengthen highschool students academic training: reading and writing, reading comprehension in a foreign language, research skills, learning and using the ICT (Information and Communication Technologies) and values development. For their part, the professors of the School of Geography of the Campus 1, "Gabino Barreda", worked on the reading and writing skills to learn Geography acfording to the current educational program. Therefore, this paper shows a conceptual overview of the importance of the literacy skills in the training of the 
students and the outcome of a survey applied to a sample of students of the campus 1 of the ENP related to their reading and writing habits and practices that they develop, both in Geography and in other subjects, which denote challenges to urgently strenghten skills such as reading, writing, and looking for information.

Keywords: Highschool education, reading and writing habits, teaching Geography

\section{Introducción}

La población del siglo XXI accede a diversos tipos de escritos a través de múltiples dispositivos como los smartphones, las tabletas, computadoras, sin dejar a un lado las publicaciones impresas como libros, periódicos y revistas. Para Cassany (1999) desde el hogar, la escuela o el trabajo tenemos acceso a diversos textos escritos y orales; incluso estos últimos los escuchamos en los medios de comunicación como discursos, entrevistas, debates y, la mayoría de ellos, han sido previamente redactados mediante guiones, scripts u otras formas escritas.

Ante este escenario, la escuela enfrenta un reto mayor para enseñar a leer y escribir. Así para Cassany (1999) anteriormente era suficiente con tener estudios de bachillerato, pues la preparación de los jóvenes en ese nivel los inicializa para la abundante cantidad de textos académicos y científicos que consultarán en sus estudios universitarios; pero hoy en día eso no basta. Es necesario, desde la educación básica diseñar estrategias didácticas adecuadas para formar en alumnas y alumnos el hábito de leer y en especial enseñar a escribir.

Dentro de este contexto, los siete docentes ${ }^{\mathrm{i}}$ que integran el Colegio de Geografía del plantel 1 "Gabino Barreda" de la Escuela Nacional Preparatoria (ENP) de la Universidad Nacional Autónoma de México (UNAM) desarrollaron el tema la "Lectura y escritura de textos para aprender a pensar" durante su Seminario de Análisis y Desarrollo de la Enseñanza (SADE, 20152016). Su interés por ese tema fue motivado por las dificultades de comprensión que han mostrado los alumnos de geografía al momento de leer diferentes textos o bien al redactarlos de forma estructurada y analítica con base en la búsqueda de información en diferentes fuentes.

Es así como el presente trabajo tiene como objetivo mostrar un panorama sobre la forma en que se trabaja la lectura y escritura en la asignatura de Geografía, a partir del análisis bibliográfico 
y los resultados de una encuesta diseñada y aplicada a alumnas y alumnos de cuarto y sexto año (área 3) del plantel para conocer sus prácticas de escritura, lectura y uso de internet que realizan en las asignaturas correspondientes.

\section{Materiales y métodos}

La primera parte del presente trabajo se sustenta conceptualmente a partir de la consulta bibliográfica sobre aspectos comunicativos y educativos sobre el uso de la lectura y la escritura para elaborar textos académicos en el ámbito escolar. Un estudio equivalente se puede consultar en Enríquez, Fajardo y Garzón (2015) en relación con los hábitos y técnicas de estudio de estudiantes universitarios. Mientras en la segunda parte, se exponen los resultados generales de una encuesta aplicada entre el 11 y el 22 de abril de 2016 a una muestra de alumnas y alumnos de $4^{\circ}$ y $6^{\circ}$ año de las asignaturas de Geografía y Geografía Económica del Plantel 1 "Gabino Barreda" de la ENP de la UNAM. El tamaño de la muestra fue de 180 estudiantes de cuarto año y 116 de sexto año (Estos últimos sólo fueron alumnos del área 3 de Ciencias Sociales).

Cabe señalar que en ese plantel se tenían, aproximadamente, 1300 alumnos en la asignatura de Geografía, repartidos en 26 grupos (14 en el turno matutino y 12 en el turno vespertino). La muestra para esta encuesta representó el 14\% del total de alumnos. Por su parte, la asignatura de Geografía Económica, que forma parte del área propedéutica de Ciencias Sociales, estaba conformada por 390 alumnos repartidos en 8 grupos (4 en cada turno). En este último caso, la muestra para la encuesta realizada representó el 30\% de los alumnos del área correspondiente.

Posteriormente, se realizó un análisis cuantitativo de frecuencias para explorar y conocer, de forma preliminar, determinados hábitos y prácticas sobre la escritura y la lectura de los estudiantes que cursan asignaturas geográficas en el plantel. La encuesta incluyó 26 preguntas, tanto de respuestas breves como abiertas, las cuales se organizaron en cinco secciones: lectura, escritura, hábitos de lectura, utilización de internet y prácticas de lectura. Tal encuesta fue diseñada por las profesoras y profesores del Colegio de Geografía con base en su experiencia personal y la Encuesta Nacional de Lectura y Escritura 2015 realizada por el Consejo Nacional para la Cultura y las Artes (CONACULTA). Como anexo a este trabajo se incluye la encuesta aplicada. 


\section{La lectura y escritura como prácticas de comunicación en la educación}

El proceso de enseñanza-aprendizaje requiere de una práctica continúa de la lectura y escritura, pero es claro que se debe enseñar a hacerlo. Generalmente los docentes dan por hecho que los estudiantes tienen estas habilidades y les asignamos una serie de tareas para que estos últimos las realicen. Algunos docentes explican brevemente los lineamientos del trabajo que requieren, pero falta ese acompañamiento para lograr resultados más eficientes. Para Serrano, Duque y Madrid, (2012) es necesario una alfabetización académica para leer y escribir, así como una especialización en el área de estudio, con ello se puede escribir para aprender y poder construir el conocimiento.

La lectura es una manera de iniciar esta alfabetización y especialización académica, sin embargo, algunas investigaciones han demostrado que los alumnos tienen serios problemas en la comprensión de textos, como para organizar la información leída, además de dificultades para identificar las ideas principales y desinterés hacia una práctica lectora, lo cual genera poca capacidad de interpretación y argumentación. (Uribe-Álvarez y Camargo-Martínez, 2011). El aprendizaje de la lectura y la escritura de textos es una tarea relevante que requiere esfuerzo, tiempo y práctica pues no ocurre de manera natural. Es conveniente incorporar la enseñanza formal de la lectura y la escritura de diferentes géneros propios de cada disciplina.

El lenguaje implica cuatro modalidades: hablar, escuchar, leer y escribir. Las primeras se hacen cotidianamente y las segundas donde más se desarrollan son en el ámbito escolar, sin embargo, como señala Barbosa y Peña (2010) estas modalidades del lenguaje son interdependientes y deben tratarse de una manera integrada. En la lectura se establece un dialogo entre el escritor y el lector, este último hace uso de los conocimientos previos para interpretar variadas formas y significados distintos que encuentra en el texto y poder, posteriormente, plasmarlo por escrito (Barbosa y Peña, 2010).

El alumno aprende a escribir escribiendo. En la escuela, si bien los alumnos practican la escritura, lo hacen con los siguientes fines: tomar apuntes, hacer un trabajo monográfico y contestar un examen. La propuesta de Cassany (1999) es que los alumnos hagan composiciones de situaciones auténticas de algún problema de su comunidad, con ello se logra hacer de la escritura un instrumento didáctico que favorezca el aprendizaje, a través de la implementación de proyectos, 
donde existe un mayor énfasis en la actividad comunicativa, que requiere de las siguientes fases para su desarrollo: a) preparación, b) realización y c) evaluación.

Para lograr lo anterior es necesario cambiar de paradigma, pues generalmente la escritura solo se ha usado en el ámbito académico para la evaluación, y es necesario que a través de la escritura se creen, descubran nuevas ideas y se desarrolle el pensamiento crítico. Lo anterior requiere que el docente no únicamente juegue un papel de corrector de estilo u ortografía, sino que sea un lector que enseñe a sus alumnos que la escritura es una manera de establecer puentes de comunicación. Asimismo, el docente debe generar actividades que involucren a los alumnos para investigar a través de la lectura de un tema específico, los elementos que debe tomar en cuenta para una mayor comprensión y poder crear un escrito que le sirva para comunicarse con los demás.

El desarrollo de habilidades para le lectura y la escritura ofrecen ventajas en el proceso de enseñanza-aprendizaje, favorecen la creatividad, el pensamiento crítico y mejoran las formas de comunicación entre los estudiantes. Por su parte, también se tienen desventajas porque no todos los alumnos han desarrollado esas habilidades comunicativas $\mathrm{y}$, a muchos de ellos, hay que motivarlos a realizar un mayor esfuerzo y dedicación. Además, se debe fomentar en ellos la importancia de releer y reescribir sus borradores de textos o trabajos.

\section{La integración de la lectura y la escritura en los textos académicos del ámbito escolar}

La redacción de un texto académico en el ámbito escolar por parte del alumno preparatoriano requiere considerar sus conocimientos previos para la lectura y la escritura, así como de un asesoramiento por parte de sus profesoras y profesores de las distintas disciplinas que aprende durante su paso por la educación media superior.

En principio, el docente debe sensibilizar al alumno sobre la trascendencia de leer y escribir de forma correcta, explicándole que su importancia radica en que como universitarios tienen que irse "entrenando" en las actividades de lectura y escritura - en el nivel medio superior - para desarrollarlas de forma más eficiente en sus estudios superiores al momento de realizar sus tareas, investigaciones o trabajos de tesis, tesinas o informes académicos para titularse. 
Además, se requiere la toma de conciencia de que cuando se revisan textos académicos, la lectura y la escritura forman parte de una unidad indisoluble y necesaria para escribir de forma correcta. Sin embargo, para fines de entendimiento y por cuestiones de método, el docente tendrá que explicarles a sus alumnos de una forma diferenciada - pero interrelacionada - tanto la lectura, por un lado, así como la escritura, por el otro. En este contexto, se tendrán que tomar en cuenta varios aspectos.

Al asumir que la lectura es una actividad que pone en relación directa al lector con sus conocimientos previos y con aquellos que lee del especialista; un lector eficiente tendría que desarrollar sus habilidades cognoscitivo-lingüísticas para lograr una escritura fluida, clara y con buena organización de ideas (Barbosa y Peña, 2010).

Por su parte, Fernández, Izuzquiza, y Laxalt (2004) plantean que en el proceso de enseñar a leer a los estudiantes universitarios

- Aprender a leer es un proceso que se amplía y enriquece constantemente.

- Leer es un proceso que se prolonga durante toda la vida, en el que el lector construye el sentido del discurso escrito haciendo uso de sus competencias lingüísticas y de su experiencia.

- Leer para estudiar en la universidad significa empezar a compartir las interpretaciones propias de una comunidad específica de lectores. Es una responsabilidad indelegable de la educación universitaria, en el marco de las instituciones, hacerse cargo de la enseñanza del abordaje de textos complejos. Es fundamental que se asuma el compromiso de desarrollar acciones institucionales destinadas a enseñar a los alumnos los modos de leer e interpretar los textos propios de las comunidades académicas (Fernández, et al. 2004: 99-100).

Con respecto a la escritura, Barbosa y Peña (2010) mencionan que es una actividad social más allá de la apariencia puramente académica con la que comúnmente se le suele identificar, ya que para su elaboración se toman en cuenta (aunque a veces los lectores no lo sepan) aspectos antropológicos, sociales, cognitivos, pragmáticos y lingüísticos, que aunados a los contextos 
particulares y sociales de los lectores, la interpretación de los textos y sus significados también estarán en relación directa con los propios significados que la comunidad quiere darles.

Los estudiantes que aprenden a leer y escribir de una manera más metódica y organizada tendrán que fijarse en la estructura de los textos, analizarlos (tanto en contenido como en forma) para que ellos aprendan, posteriormente, a hacer sus propios escritos académicos. En este punto, es importante que el docente les enseñe las distintas tipologías o clasificaciones de los textos, así como del proceso didáctico que deben llevar a cabo los alumnos para empezar a elaborar los suyos.

Con relación a algunas tipologías de textos, Barbosa y Peña (2010) nos mencionan que hay textos informativos y especializados. Los primeros son generales, pudiendo ser de divulgación científica o para informarse sobre el estado del tiempo, para conocer la distancia de un lugar a otro, buscar un número telefónico, etcétera; información que puede encontrarse en mapas, diccionarios, enciclopedias o internet. Los segundos se utilizan para ampliar la información y el conocimiento sobre un tema en particular. Esta información se obtiene de los artículos y libros que hacen los investigadores del más alto nivel académico y científico. En cuanto al proceso didáctico que deben llevar a cabo los alumnos para elaborar sus propios escritos, estos autores nos señalan la existencia de tres etapas:

a) Antes de la lectura. Implica seleccionar el tema, establecer los objetivos, pensar en el lector potencial, revisar la bibliografía y seleccionar las lecturas básicas. También es necesario comentar con los compañeros y el profesor la situación social en la que se dará la exposición de la información, elegir el género textual y elaborar esquemas conceptuales sobre el tema que se va a escribir;

b) Durante la escritura, se transforman las ideas en palabras, se revisan los esquemas, se elaboran borradores y se realiza una revisión crítica de la bibliografía para descartar la menos relevante. Además, deben plantearse preguntas sobre lo leído para buscar más información complementaria, comenzar la elaboración de la estructura del texto con los conocimientos previos $\mathrm{y}$ adquiridos en las lecturas, anticipar preguntas y dudas que podrían plantearse los lectores. Finalmente, se sugiere tomar en cuenta lo que previamente se ha dicho antes de agregar más 
información, especificar con precisión lo que se escribe y volver sobre los esquemas previamente elaborados para confirmar que se escribe dentro de la línea de pensamiento planteada y

c) después de la escritura, es conveniente identificar logros, problemas textuales, inconsistencias o vacíos de información para resolverlos; suprimir o cambiar afirmaciones y opiniones; ampliar contenidos; anticipar preguntas posibles para que el lector encuentre las respuestas; comprobar el hilo conector del texto y corregir las ambigüedades.

Y en cuanto a la estructura del texto, este debe contener una Introducción (que anticipe y resuma el contenido); un desarrollo o cuerpo (que contenga el análisis e interpretación del objeto de estudio); una conclusión o cierre (donde se retomen los objetivos y los problemas planteados previamente) y las citas textuales y referencias bibliográficas (esenciales para la elaboración de un texto académico).

\section{Análisis de los resultados de la encuesta a alumnas y alumnos de cuarto y sexto año de Geografía del plantel 1 "Gabino Barreda" de la ENP}

A partir de los aspectos conceptuales y teóricos revisados en torno a la lectura y la escritura se exponen los principales resultados observados de la aplicación de la encuesta a las alumnas y alumnos de cuarto y sexto año (área 3) del plantel 1 de la ENP de acuerdo con los siguientes cinco rubros correspondientes (Véase un resumen de los resultados en el Cuadro 1).

\section{La lectura y los materiales utilizados}

- Los alumnos de cuarto año reportan que leen más en las asignaturas de Lengua, Historia y Geografía, porque en ellas les son asignadas mayor cantidad de lecturas para buscar información o para hacer un análisis de estas. Un segundo grupo de asignaturas incluye a Lógica, Orientación e Informática, en las asignaturas restantes los alumnos respondieron que leen menos. Se infiere que depende del maestro y las actividades que establezca en el desarrollo del curso para lograr que los alumnos lean más.

- En tanto, los alumnos de sexto año del área 3 leen más en Literatura y Geografía Económica, pues requieren un mayor análisis de información, la comprensión de textos o 
bien la argumentación crítica de los mismos. Un segundo grupo de asignaturas incluye a Problemas Sociales, Económicos y Políticos de México (PSEPM), Psicología y Geografía Política; mientras Introducción a las Ciencias Sociales (ICS), Higiene Mental y Sociología, junto con las asignaturas de idiomas y Contabilidad son mencionadas como las asignaturas donde leen menos. Por lo tanto, se interpreta que dependerá del docente el fomentar la lectura al proporcionarles o sugerirles leer una mayor cantidad de lecturas. En este caso, hay unas materias donde leen más y en otras no, de las cuales supondríamos deberían leer más como es ICS o Sociología.

- Para la mayor parte de las alumnas y alumnos encuestados, los profesores de Geografía y Geografía Económica diseñan actividades didácticas para leer de forma individual o grupal.

- Al comparar los hábitos de lectura entre los estudiantes de cuarto y sexto año se encontró lo siguiente: Las técnicas de lectura empleadas son el subrayado de ideas principales y palabras clave, solo que por experiencia se ha observado que la mayoría de los alumnos no aplica la técnica adecuadamente. La lectura requiere tiempo y dedicación para después de hacerla se logre un análisis del contenido, por ello se recomienda que esta técnica no se haga en la primera lectura general de reconocimiento, sino en una segunda lectura de análisis y síntesis, para ubicar las ideas principales y las palabras clave.

Tabla 1. Principales criterios y resultados de la encuesta sobre hábitos y prácticas para la lectura y escritura aplicada a estudiantes de cuarto y sexto año del Plantel 1 "Gabino Barreda" de la Escuela Nacional Preparatoria, UNAM (2016) 


\begin{tabular}{|c|c|c|}
\hline Criterios & $4^{\circ}$ Año & $6^{\circ} \mathrm{Año}(a ́ r e a)$ \\
\hline $\begin{array}{l}\text { 1. Asignaturas en las que } \\
\text { más leen }\end{array}$ & Lengua. Historia. Geografia. & $\begin{array}{lrr}\text { Geografia } & & \text { Económica, } \\
\text { Literatura } \\
\text { Política. }\end{array}$ \\
\hline $\begin{array}{l}\text { 2. Técnicas de lectura } \\
\text { empleadas }\end{array}$ & $\begin{array}{l}\text { Subrayado, ideas principales, } \\
\text { palabras clave. }\end{array}$ & $\begin{array}{l}\text { Subrayado, ideas principales, } \\
\text { palabras clave. }\end{array}$ \\
\hline $\begin{array}{l}\text { 3. Tipos de materiales } \\
\text { utilizados para leer textos }\end{array}$ & Libros, copias, computadora. & $\begin{array}{l}\text { Copias, libros, computadora, } \\
\text { teléfono inteligente. }\end{array}$ \\
\hline $\begin{array}{l}\text { 4. Asignaturas en las que } \\
\text { escriben más }\end{array}$ & Lengua, Geografia e Historia. & $\begin{array}{l}\text { Geografia económica, } \\
\text { Literatura y Psicología. }\end{array}$ \\
\hline $\begin{array}{l}\text { 5. Fuentes de información } \\
\text { para hacer tareas y } \\
\text { trabajos }\end{array}$ & $\begin{array}{l}\text { Libros impresos y digitales, } \\
\text { sitios web. }\end{array}$ & $\begin{array}{l}\text { Sitios web, libros impresos y } \\
\text { digitales. }\end{array}$ \\
\hline $\begin{array}{l}\text { 6. Tipos de escritos } \\
\text { elaborados }\end{array}$ & $\begin{array}{l}\text { Apuntes, resumen y cuadros } \\
\text { sinópticos/mapas } \\
\text { conceptuales. }\end{array}$ & $\begin{array}{l}\text { Apuntes, cuadros } \\
\text { sinópticos/mapas } \\
\text { conceptuales y resúmenes. }\end{array}$ \\
\hline $\begin{array}{l}\text { 7. Uso que le dan a } \\
\text { Internet. }\end{array}$ & $\begin{array}{l}\text { Buscar información, } \\
\text { participación en redes } \\
\text { sociales, descargar música, } \\
\text { fotos y videos. }\end{array}$ & $\begin{array}{l}\text { Buscar información, } \\
\text { participación en redes } \\
\text { sociales, descargar música, } \\
\text { fotos y videos. }\end{array}$ \\
\hline $\begin{array}{l}\text { 8. Prácticas didácticas } \\
\text { para retener y comprender } \\
\text { información. }\end{array}$ & $\begin{array}{l}\text { Apuntes, análisis de textos } \\
\text { para aclarar dudas, ideas para } \\
\text { hacer resumen, lectura } \\
\text { informativa. }\end{array}$ & $\begin{array}{l}\text { Análisis de textos para aclarar } \\
\text { dudas, apuntes, ideas para } \\
\text { hacer resumen, lectura } \\
\text { informativa. }\end{array}$ \\
\hline
\end{tabular}

Fuente: Elaboración propia con base en los tres primeros resultados de las encuestas.

- También se observó que, en los alumnos de cuarto año, la lectura la realizan generalmente en libros, mientras los de sexto año ocupan más las copias. Cabe señalar que en los primeros años de preparatoria los profesores emplean libros de texto para sus cursos, en mayor medida. Por último, se apreció un segundo grupo de alumnos que utilizan la computadora para hacer búsquedas en internet y otro más leen revistas o periódicos en el teléfono inteligente o tableta.

\section{La escritura y sus materiales empleados}

- De acuerdo con la encuesta aplicada en cuarto año el $81.7 \%$ de los alumnos les gusta escribir, mientras un $18.3 \%$ no le gusta hacerlo; pero en sexto año al $85 \%$ sí les gusta escribir, mientras un $15 \%$ no. Estos resultados obedecen al hecho de que los alumnos de 
este nivel educativo deben escribir de forma frecuente en sus asignaturas, además la mayoría lo hacen porque desarrollan mayores habilidades de escritura, las cuales les serán fundamentales para sus estudios de educación superior.

- Al comparar los porcentajes con la frecuencia empleada para escribir textos se encontró que un $64.8 \%$ de los alumnos de cuarto año lo hace de forma regular, un $18.3 \%$ de forma frecuente y el $16.9 \%$ lo hacen en menores proporciones y muy pocos mencionaron que nunca lo hacen. Por su parte, en el caso de alumnos de sexto año los porcentajes fueron del $63 \%, 24 \%$, respectivamente, y en menores proporciones hay algunos alumnos que casi o nunca lo hacen.

- En cuanto a las asignaturas de mayor lectura y escritura en ambos grados los resultados mostraron que aquellas corresponden con las áreas de Ciencias Sociales y Humanidades. Así, en el caso de las asignaturas en las cuales escriben más los alumnos, tanto de cuarto como de sexto año, manifestaron que lo hacen en las mismas asignaturas en las cuales también leen: Lengua, Geografía e Historia o bien Geografía Económica y Literatura. Un segundo grupo con menor proporción lo representan Lógica, Física, Informática y Matemáticas o bien Psicología, PSEPM y Geografía Política, según el grado escolar respectivo. No obstante, para cuarto año, en la asignatura de Orientación leen más y escriben menos; pero en sexto año, los alumnos aseveraron escribir más en Derecho que leer. Asimismo, estos alumnos escriben poco en Sociología, Higiene Mental y las asignaturas de idiomas, además se observó que, en Matemáticas, prácticamente no leen, pero si escriben más.

- En cuanto a la evaluación de los escritos elaborados por alumnos de cuarto y sexto año, se aprecia que gran parte de los docentes de Geografía sí revisan los trabajos, señalan errores ortográficos y de sintaxis o bien marcan observaciones con relación al contenido o aspectos faltantes. En el plantel, la proporción de profesores que solo sellan o firman tareas es mínima. Además, la encuesta mostró que los profesores deben trabajar más con la corrección de los escritos, así como dar un seguimiento de tales correcciones para ver el grado de avance de los alumnos. En este último caso, la experiencia docente muestra que 
la mayor dificultad para cubrir ese punto depende del número de alumnos del grupo, pues en promedio, se tienen cerca de 50 a 60 alumnos.

- Por su parte, alumnas y alumnos de ambos grados emplean Tecnologías de la Información y la Comunicación (TIC) de forma cotidiana para realizar sus tareas o trabajos Así, la mayoría obtiene la información de sitios web de instituciones u organismos y de libros impresos, tal como sucede con sus libros de texto o de aquellos que consultan en la biblioteca del plantel. Pero, otra proporción cada vez más creciente emplea libros digitales, videos de YouTube, Blogs, Sitios Web diversos como Wikipedia o Buenastareas. Otros más emplean revistas y periódicos impresos y en menor proporción de forma digital. También fue notorio apreciar que las redes sociales no las usan tanto para hacer trabajos y tareas, aunque si lo hacen para comunicarse entre sí.

- En relación con los escritos elaborados por los alumnos de Geografía en ambos grados se observó que frecuentemente toman apuntes, redactan resúmenes, reportes de lecturas, de videos o elaboran cuadros sinópticos y mapas conceptuales. También se evidenció que, a diferencia de los alumnos de cuarto año, los alumnos del último año de la preparatoria contestan menos cuestionarios, pues realizan más investigaciones y se promueve el análisis e interpretación de mapas o cuadros estadísticos.

\section{Hábitos de lectura}

- La preparatoria promueve la lectura de diversos textos tal como lo señalan los resultados de la encuesta, pues una gran proporción de alumnas y alumnos coincide que leen más a diferencia de la educación secundaria. Incluso, el 95.6\% de los alumnos de cuarto año les es fácil leer, pero en los de sexto año, el porcentaje solo fue del 90\%, lo cual muestra la necesidad de favorecer más el hábito de la lectura.

- La frecuencia con la que leen los alumnos de cuarto año es la siguiente: el 31.7\% lo hace diario, el $37.8 \%$ ocasionalmente, $19.4 \%$ hasta dos veces por semana, en tanto un $11.1 \%$ lo hace una vez a la semana. Lo que quiere decir que el $88.9 \%$ de los alumnos si tiene el hábito 
de la lectura. Mientras, para los alumnos de sexto año esa frecuencia es variable: el $40 \%$ lo hace de forma ocasional, un $30 \%$ lo hace hasta dos veces por semana, en tanto un $21 \%$ lo hace diariamente y solo un $9 \%$ lee una vez a la semana.

- En relación con los hábitos empleados para leer los estudiantes, en general de ambos años escolares, manifestaron los siguientes aspectos: 1) La mayoría lee rápido y frecuentemente no comprenden lo que leen, pues su concentración es mínima debido a diversos distractores. 2) Un segundo grupo de alumnos afirmó que sí se concentran al leer, no son impacientes y una buena parte de ellos terminan los libros que leen. Por lo tanto, es evidente que a los alumnos de preparatoria les hacen falta hábitos y técnicas de lectura.

- Finalmente, alumnas y alumnos manifiestan leer más libros y ocasionalmente periódicos, revistas y comics, pero la experiencia demuestra la necesidad de fomentar la lectura de periódicos, pues los alumnos reciben mensajes de texto en sus dispositivos con las noticias relevantes del día, pero éstas contienen información breve y en ocasiones muy superficial.

\section{Utilización de internet}

- Los resultados de cuarto año indican que el $73 \%$ de los estudiantes encuestados afirmaron emplear internet para realizar sus tareas y lo utilizan diariamente; en tanto un $25.3 \%$ señaló usar la red algunas veces, pero lo hacen gran parte del tiempo a la semana, solo el $1.7 \%$ manifestó no usar internet. Por su parte, en el caso de sexto año, el 70\% de los alumnos emplean internet diariamente para hacer tareas y un $30 \%$ usan la red algunas veces a lo largo de la semana.

- En relación con la duración del tiempo dedicado a usar internet, los alumnos de cuarto año $(58.6 \%)$ y los alumnos de sexto año (52\%) lo utilizan de media a una hora; mientras $23 \%$ de cuarto año y $30 \%$ de sexto emplean más de cinco horas y sólo un $7.3 \%$ de los alumnos de cuarto año lo usan todo el día. Por lo tanto, los alumnos pasan un buen tiempo del día conectados a internet para realizar diversas actividad escolares y personales, por lo que deben leer, más no necesariamente lo emplean para escribir.

Revista RedCA octubre 2020 - enero 2021

ISSN: 2594-2824 Vol. 3 Núm. 8 
- Asimismo, se observó, en términos generales para ambos grupos, que la mayoría emplea internet para buscar información escolar o bien participar en las redes sociales y chatear. En menor proporción lo usan para descargar música, fotos o videos o para leer libros, periódicos o revistas. Finalmente, un porcentaje menor señaló que lo usa para jugar, lo cual creemos que no es del todo cierto, pues por experiencia sabemos que un porcentaje mayor de alumnos usa internet para jugar. Por ello es necesario diseñar actividades para mantenerlos ocupados con un menor uso del celular y crear estrategias educativas para utilizar las TIC en clase.

- También, una tendencia observada de los alumnos, tanto de cuarto como de sexto año, es que consultan internet para hacer su tarea, por lo que se usan menos la biblioteca. Además, la encuesta mostró el uso importante del buscador Google y sitios académicos con terminación edu., org., gov, etc. En menor medida algunos mencionaron emplear sitios como Wikipedia, Buenastareas, monografías, entre otros. Asimismo, se observó un gran desconocimiento sobre los servicios gratuitos ofrecidos por la Dirección General de Bibliotecas de la UNAM para obtener una variedad de información de calidad, pero que no se usa por desconocimiento. En este sentido, los maestros debemos proporcionarles información para facilitar su acceso.

- En relación con los hábitos y técnicas empleadas para hacer trabajos y buscar información en medios electrónicos, la encuesta mostró que el $64.9 \%$ de los alumnos de cuarto año leen, subrayan palabras clave para después escribir sobre el tema y citan la fuente consultada; en tanto los alumnos de sexto año alcanzaron un $76 \%$ en este rubro. Mientras, un 30\% (de cuarto año) y un 24\% (de sexto año) leen, copian y pegan la información del tema correspondiente a la tarea y citan la fuente utilizada. La experiencia muestra que la mayoría de la alumnas y alumnos hace un uso incorrecto para citar la información, pues generalmente cortan y pegan incurriendo en problemas de plagio, por ello la labor docente debe encaminarse para hacerlos responsables y respetuosos del trabajo intelectual de los demás, una manera de hacerlo es enseñándoles a citar las fuentes de consulta.

\section{Prácticas de lectura}


- Finalmente, la encuesta aplicada a los estudiantes preparatorianos exploró algunos hábitos, técnicas o intereses relacionados con la lectura y la escritura de temas relacionados con la Geografía.

- En el caso de las prácticas didácticas empleadas para retener y comprender la información geográfica se observó, en términos generales para ambas asignaturas, que la mayoría de los alumnos afirman realizar apuntes y resúmenes por cuenta propia de lo que leen. Otro sector importante analiza lo que no entiende y busca dudas en diversos medios impresos y digitales para comprender la información leída. Un tercer grupo hace lista de ideas fundamentales de lo que lee y redacta un resumen de esta; pero en menor proporción, los alumnos realizan cuadros sinópticos de sus lecturas o bien simplemente leen y confían en su memoria para recordar la información principal.

- Por su parte, los temas geográficos que les hubiera gustado que el profesor les recomendará leer más en la asignatura de Geografía para cuarto año fueron, en primer lugar, los movimientos terrestres, husos horarios y mapas; en segundo lugar temas de Geografía Económica y Geografía Política, globalización, países desarrollados y en desarrollo; en tercer lugar sobre la historia del pensamiento geográfico; mientras temas como la dinámica de la Tierra, tectónica global, formas de relieve se ubicaron en cuarto lugar, y en quinto mencionaron los temas del ciclo del agua, atmósfera, biosfera y los problemas ambientales.

- Mientras, para los alumnos de sexto año los temas geográficos para leer que esperaban por recomendación del profesor se ubican en el ámbito de la Geografía Humana (Geografía de la Población, Geografía económica y Geografía política), lo cual reafirma el interés de los alumnos de sexto año sobre aspectos sociales propios de esta área. Aun así, un grupo importante de alumnos manifestó interés sobre temas relacionados con la atmosfera, la hidrosfera y la biosfera o bien sobre la Tierra como astro. Y los temas con menor interés se relacionan con la historia del pensamiento geográfico o la dinámica interna y externa de la corteza terrestre.

Revista RedCA octubre 2020 - enero 2021

ISSN: 2594-2824 Vol. 3 Núm. 8 
- Por otro lado, el 61\% de los alumnos (de cuarto año) y el 86\% (de sexto año) señalaron que durante el curso hubo temas que les llamaron la atención para investigar más mediante lectura voluntaria; pero un 39\% (de cuarto año) y un 14\% (de sexto año) no le interesó profundizar en los temas. Por ejemplo, en la Figura 1 se observan los temas específicos de la Geografía que despertaron más interés en los alumnos del área 3 y en la cual destacan los relacionados con los climas, la población, el proceso económico y las migraciones. También manifestaron interés por una gama amplia de temas específicos, pero de forma más bien individual, como tectonismo, el Medio Oriente, la historia de la Geografía, la organización política del mundo, la colonización, las fronteras o bien sobre países como China, Japón, India, entre otros.

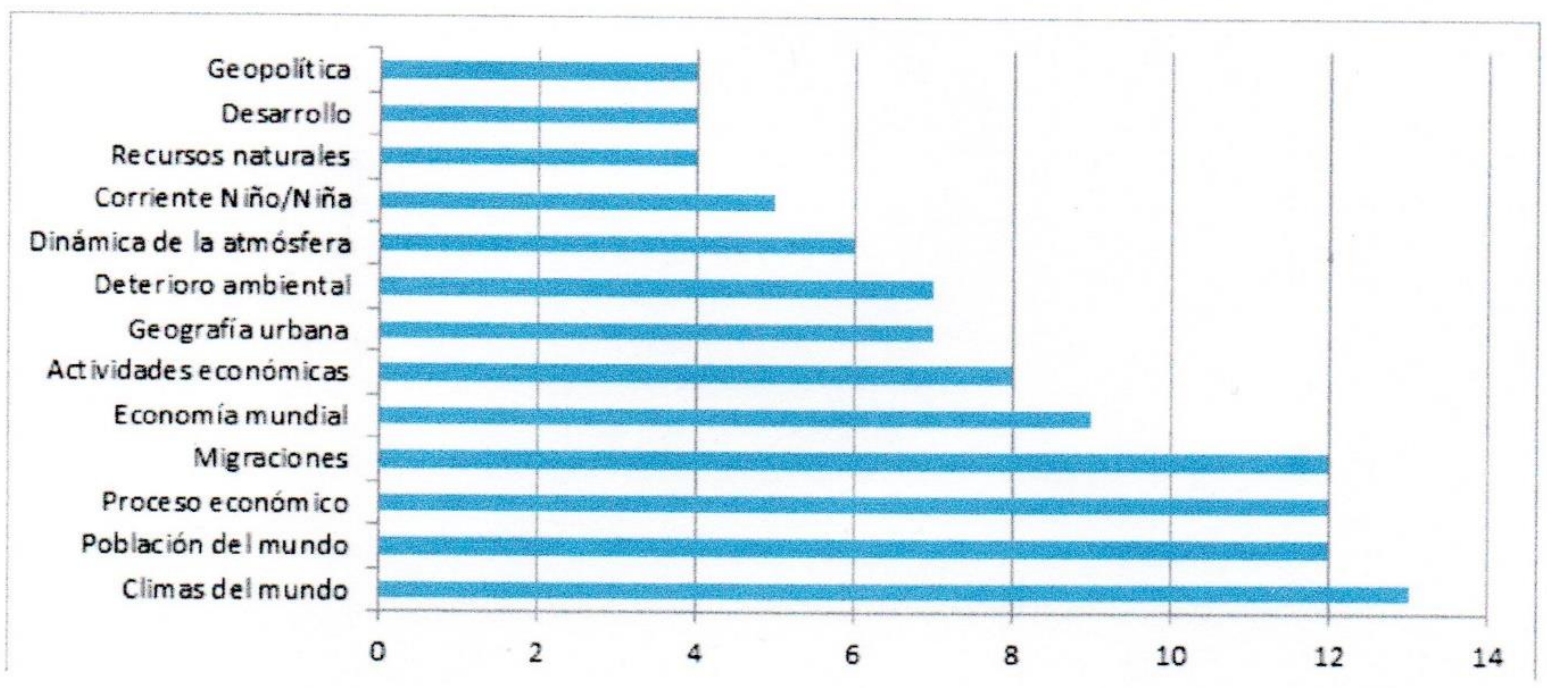

Figura 1. Temas geográficos que despertaron más interés en alumnas y alumnos de sexto año del área 3 del Plantel 1 "Gabino Barreda" de la Escuela Nacional Preparatoria, UNAM (2016). Fuente: Elaboración propia con base en la encuesta aplicada a alumnos del plantel.

- En otro rubro, un $78.6 \%$ de alumnos de cuarto año y un $84 \%$ de sexto año afirmaron leer por su cuenta para comprender los temas abordados, completar sus apuntes de clase, no quedarse con las dudas y preparar sus exámenes; en tanto el $21.4 \%$ (de cuarto año) y el 16\% (de sexto año) manifestaron no leer de más, porque les da flojera, tienen apatía, les falta tiempo o no se acuerdan de hacerlo.

Revista RedCA octubre 2020 - enero 2021 ISSN: 2594-2824 Vol. 3 Núm. 8 
- Para finalizar la encuesta las alumnas y alumnos autoevaluaron sus prácticas de lectura en una escala del 1 al 10, en espera de que al leer tengan una idea general del texto para comprenderlo y retener la información básica. Así, los alumnos de cuarto año se evaluaron con puntajes de 8, 7, 9 y 10 mayoritariamente; mientras los alumnos de sexto año obtuvieron puntajes de 8,7 y 9 principalmente, lo cual denota que son lectores de buenos a regulares.

\section{Conclusiones}

A través de este ensayo se constató la necesidad de fomentar la lectura y escritura de textos para aprender a pensar en los estudiantes de la ENP.

El marco conceptual fundamentó aspectos indispensables para que los profesores reflexionen sobre la importancia de superar actividades básicas de lectura y escritura y transitar hacia el desarrollo de textos en los cuales los estudiantes lean y escriban de una manera crítica y reflexiva. Por ello, los alumnos deben desarrollar habilidades de lectura de textos, búsqueda y compilación de información, así como la estructuración y escritura de textos descriptivos y argumentativos relacionados con diversos temas de los programas de las asignaturas correspondientes.

La encuesta aplicada permitió obtener algunas observaciones preliminares relacionadas con los hábitos y las diversas prácticas de lectura y escritura que los alumnos de cuarto y sexto años realizan en sus asignaturas, y en particular en las de Geografía. Tales resultados ofrecen información para que los profesores las tomen en cuenta en el diseño de estrategias de enseñanza y aprendizaje adecuadas para la promoción de la lectura y escritura entre los estudiantes. En términos generales, los alumnos leen y escriben más en las asignaturas de las áreas de Ciencias Sociales y Humanidades como resultado de las actividades didácticas que las profesoras y profesores plantean para el desarrollo de los temas de sus respectivas asignaturas.

Al momento de leer los alumnos subrayan ideas principales y rescatan palabras o ideas que anotan en su cuaderno de notas. Además, emplean de forma preponderante copias, libros, computadora o sus teléfonos inteligentes, sobre otras publicaciones como las revistas y periódicos, 
lo cual es propio de los estudiantes preparatorianos, pues están poco habituados a leerlos, salvo para revisar temas de su interés.

En cuanto a la escritura, los estudiantes encuestados les gusta escribir, aunque no lo hacen de forma frecuente, ni espontánea, salvo para tomar apuntes o realizar sus tareas escolares como reportes, organizadores gráficos o resolver cuestionarios; no obstante, los alumnos de sexto año afirman realizar investigaciones o bien análisis e interpretación de mapas y cuadros estadísticos. Por su parte, los resultados de la encuesta muestran que las alumnas y alumnos escriben más en las asignaturas de Ciencias Sociales y Humanidades, tal como sucedió con la actividad lectora; además, sus escritos son revisados por los docentes quienes les corrigen ortografía y sintaxis, principalmente, pero reconocen la falta de continuidad para revisarles las correcciones de sus trabajos, como resultado de la saturación de alumnos en los grupos.

Al momento de escribir, los estudiantes consultan diversas fuentes digitales en internet o bien revisan sus libros de texto, que son más comunes de usar en el cuarto año de la preparatoria. También visitan la biblioteca del plantel para revisar bibliografía o realizar sus trabajos. No obstante, se aprecia un incremento de consultas específicas en internet para obtener información, tal como sucede con los videos, revistas digitales o blogs.

En cuanto a sus hábitos de lectura se apreció que un porcentaje superior al $80 \%$ están acostumbrados a leer, aunque no lo hacen diariamente. Leen libros y revistas, principalmente. Asimismo, cuando leen lo hacen rápido, según externo una mayoría de alumnos, en tanto, un porcentaje menor manifestó realizar una lectura más lenta, pues requieren concentrarse para lograr una mayor comprensión.

En relación con el uso de internet, aproximadamente el 72\% de los estudiantes encuestados afirmo usarlo diariamente para hacer sus tareas o mantener comunicación y emplean buscadores como Google, así como algunos sitios informativos como Wikipedia o monografías; mientras un porcentaje menor descarga información como videos, fotografías o música. También, la encuesta constató que los alumnos cortan y pegan información, citan documentos, pero no siempre redactan de forma autónoma sus textos. La encuesta finaliza con prácticas de lectura y en este caso los alumnos leen, resumen textos, buscan las dudas que tienen en diversos medios y estudian para sus 
exámenes. Asimismo, tienen interés por temas geográficos, en particular sobre aspectos sociales, económicos, climáticos y ambientales.

Por consiguiente, los aspectos expuestos en este trabajo ofrecen una mirada particular sobre los hábitos y las prácticas que realizan los estudiantes encuestados de un plantel de la ENP, las cuales se acercan a las halladas en otros trabajos de investigación educativa. Por tanto, se fortalece la necesidad de que los docentes diseñen estrategias didácticas significativas en el ámbito de la lecto-escritura en el proceso formativo de los alumnos preparatorianos.

\section{Referencias bibliográficas}

Barbosa Peña, F. y Peña González, F. (2010). Integración lectura y escritura en la composición de textos académicos. Consideraciones desde la teoría y la práctica. Revista Educere, 14(48). pp. 53-61. Recuperado de: http://www.redalyc.org/articulo.oa?id=35616720006

Cassany, D. (1999). Construir la escritura. Barcelona: Paidós.

Enríquez Villota M. F., Fajardo Escobar M., Garzón Velásquez F. (2015). Una revisión general a los hábitos y técnicas de estudio en el ámbito universitario. Psicogente, 18(33), 166-187 Recuperado de: https://www.redalyc.org/pdf/4975/497551992015.pdf

Fernández, G., Izuzquiza, M. V. y Laxalt, I. (2004). El docente universitario frente al desafío de enseñar a leer. En P. Carlino (Coord.), Textos en Contexto. No. 6, 95-110. Buenos Aires: Lectura y Vida/Asociación Internacional de Lectura.

Secretaria de Cultura (2016). Encuesta Nacional de Lectura y Escritura 2015. México: CONACULTA. Recuperado de: https://observatorio.librosmexico.mx/encuesta.html 
Serrano, M. S., Duque, Y., Madrid, A. (2012). Prácticas de escritura académica en la universidad: ¿reproducir o transformar? Educere. 16 (53), 93-108. Recuperado de: http://www.redalyc.org/articulo.oa?id=35623538011

Uribe-Álvarez, G. y Camargo-Martínez, Z. (2011). Prácticas de lectura y escritura académicas en la universidad colombiana. Magis. Revista Internacional de Investigación en Educación, 3(6), 317-341. Recuperado de: http://www.redalyc.org/articulo.oa?id=281021734005

Nota: Además de los autores del presente trabajo, también colaboraron en el SADE 2015-2016 los profesores María de las Mercedes Moreno Maldonado, José R. Balanzario Zamorate y Carlos Ángel Valdivia Martínez del Colegio de Geografía del plantel 1 de la ENP de la UNAM. 
UNIVERSIDAD NACIONAL AUTÓNOMA DE MÉXICO

ESCUELA NACIONAL PREPARATORIA N. 1 "GABINO BARREDA"

COLEGIO DE GEOGRAFIA SADE LOCAL 2015-2016

Encuesta para alumnos de la prepa 1 Grupo

\section{LECTURA}

1. Anota las materias dónde lees más

*Puedes anotar más de una materia.

2. En la clase de geografía, tu profesor diseña actividades didácticas para leer de forma individual o grupal ( ) SI ( ) No

3. Cuando leen textos (libros, artículos, copias, etc....) en la clase de geografía:

( ) Subrayas ideas importantes

( ) Colocas marcas en los márgenes de los textos

( ) Anotas palabras clave en los márgenes de los textos

( ) Rescatas ideas importantes y las anotas en un cuaderno/ficha de lectura/o de forma digital.

( ) No subrayas, ni marcas textos.

*Puedes marcar más de una opción.

4. Jerarquiza en orden creciente el tipo de materiales empleados para leer textos escritos en la materia de Geografía

$\begin{array}{lll}(\quad) \text { Copias } & (\quad) \text { Computadora/laptop } \\ (\quad) \text { Libros } & (\quad) \text { Periódicos } \\ (\quad) \text { Revistas } & (\quad) \text { Tabletas digitales } \\ (\quad) \text { Teléfono inteligente (Smartphone) } \\ (\quad) \text { Otros }\end{array}$

\section{ESCRITURA}

5. ¿Te gusta escribir? ¿Por qué?

6. ¿Con que frecuencia realizas un escrito?

$\begin{array}{ll}\text { Mucha ( ) } & \text { regular ( ) } \\ \text { poca ( ) } & \text { nunca ( ) }\end{array}$

7. Anota las materias dónde escribes más

*Puedes anotar más de una materia

8. Cuando entregas trabajos o tareas escritas en geografia, tu profesor(a) los evalúa y

( ) Te señala errores ortográficos y gramaticales.

( ) Te escribe aspectos faltantes como datos personales, bibliografia, etc.

( ) Te marca observaciones de contenido.

( ) Solo te firma o sella.

( ) Te pide corregir y devolverlo después (reescribir).

*Puedes marcar más de una opción

9. Cuando redactas trabajos o tareas escritos en geografía, la información la obtienes principalmente de

( ) Libros impresos

( ) Revistas/periódicos impresos

( ) Libros digitales

( ) Revistas/periódicos digitales

( ) Sitios web de instituciones u organismos

( ) Blogs

( ) Sitos web diversos como wikipedia, buenastareas, etc

Revista RedCA octubre 2020 - enero 2021 ISSN: 2594-2824 Vol. 3 Núm. 8 
( ) Videos/documentales en Youtube

( ) Redes sociales

( ) Otros

*Puedes marcar más de una opción.

10. Qué tipo de escritos realizas de forma frecuente en las clases de geografía

( ) Ensayos ( ) Resúmenes

( ) Cuadros sinópticos/mapas conceptuales

( ) Apuntes ( ) Cuentos/historietas

( ) Reportes de lecturas ( ) Cuestionarios

( ) Investigaciones libros/internet

( ) Reseñas de libros o eventos

( ) Reportes de videos o documentales

( ) Análisis e interpretación mapas/cuadros

( ) Otros

*Puedes marcar más de una opción.

\section{HÁBITOS DE LECTURA}

\section{Para ti ¿es fácil o difícil leer?}

( ) Fácil

( ) Dificil

12. ¿Con qué frecuencia lees?

( ) Ocasionalmente

( ) Una vez a la semana

( ) Dos veces a la semana

13. Cuál de las siguientes opciones se te presentan cuando lees un libro? Anota Si o No según corresponda en cada caso

Leo muy despacio

$\overline{\mathrm{A} \text { veces no comprendo o entiendo lo que leo }}$

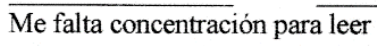

Soy impaciente para leer

Dejo libros a la mitad

\section{Desde mi ingreso a la Preparatoria}

( ) Leo más

( ) Leo igual

( ) Leo menos

\section{Actualmente leo:}

\section{Siempre Ocasionalmente}

Periodicos

Revistas

Libros

Cómics o historietas

\section{UTILIZACIÓN DE INTERNET}

(Subraya la respuesta correcta)

16. Utilizas internet para resolver tus tareas

a) $\mathrm{Sí}$

b) Algunas veces

c) No (Pasa a la pregunta 22)

d) Nunca (Pasa a la pregunta 22)

17. ¿Con qué frecuencia utilizas internet?

a) Diario

b) Varias veces a la semana

c) Una vez a la semana

d) Ocasionalmente

18. ¿Cuánto tiempo te conectas en cada ocasión?

a) Menos de media hora

b) Entre media hora y una hora

c) Más de cinco horas

d) Todo el día

19. ¿Cuáles son las principales razones por las que usas internet?

a) buscar información y estudiar

b) participar en redes sociales y chatear

c) descargar música, fotos y videos

d) leer libros, periódicos y revistas

Revista RedCA octubre 2020 - enero 2021 ISSN: 2594-2824 Vol. 3 Núm. 8 
20. Cuándo buscas información para resolver tus tareas, lo haces en:

a) Dirección General de bibliotecas UNAM

b) Google académico o instituciones gubernamentales

c) En páginas con dominio o terminación edu., org., gov, net.

d) Wikipedia, Buenas tareas, Monografias.com, Yahoo respuestas, El rincón del vago

*Puedes subrayar más de una opción.

21. Cuándo elaboras un trabajo y obtienes información en medios electrónicos, haces lo siguiente:

a) Lees y subrayas las palabras clave para después escribir sobre el tema. Citas la fuente de consulta completa.

b) Lees, copias y pegas la información sobre el tema, citas la URL de la fuente de consulta.

c) No lees solo buscas palabras sobre el tema, copias y pegas sin citar las fuentes de consulta.

d) Buscas un trabajo sobre el tema y solo copias $\mathrm{y}$ pegas.

\section{PRACTICAS DE LECTURA}

22. ¿Qué temas de la Geografía (Física o Humana) te gustaría que el profesor te hubiera recomendado leer?

a) De la Historia del pensamiento geográfico.

b) De la Tierra como astro (movimientos de la Tierra, Traslación, Rotación, Husos Horarios, Mapas, etc.)

c) De la dinámica interna de la Tierra (capas de la Tierra, Tectónica Global, Formas del Relieve, etc.).

d) Del ciclo del agua y su relación con la atmósfera, el océano y la biosfera, así como de sus problemas ambientales.

e) De temas de Geografía de la Población, de Geografía Económica o de Geografía Política.

*Puedes subrayar más de una opción.
23 Para retener y comprender la información que lees, qué prácticas didácticas llevas a cabo para obtener los resultados deseados de retención y comprensión?

a) Realizo apuntes y resúmenes por cuenta propia acerca de lo que leo.

b) Analizo lo que no entiendo y busco en diccionarios, enciclopedias, libros o internet las dudas que me genera la lectura

c) Hago una lista de las ideas fundamentales de la lectura para, después, redactar un resumen de la misma

d) Llevo a cabo cuadros sinópticos que me permiten visualizar la generalidad de la lectura.

e) Simplemente leo y confio en mi memoria.

24 ¿Durante el curso de Geografía, te llamó la atención un tema o una serie de temas que propiciaron la lectura voluntaria? ( ) SI ( ) No

¿Cuál o cuáles fueron esos temas?

25 Cuándo tienes alguna duda sobre lo que te enseñan tus profesores de la prepa, y si a pesar de que tus maestros te vuelvan a explicar y no logras entender ilees por tu parte y completas tus apuntes de clase de forma voluntaria?

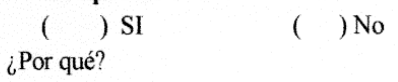

26 En una escala del 1 al 10 , ¿con qué número te evaluarías en tus prácticas de lectura? Toma en cuenta que una vez hecha la lectura, debes tener la idea general del texto para poder comprenderla $y$ retenerla de forma satisfactoria.

$\begin{array}{llll}1 & 4 & 7 & 10 \\ 2 & 5 & 8 & \\ 3 & 6 & 9 & \end{array}$

Gracias por tu participación

Revista RedCA octubre 2020 - enero 2021 ISSN: 2594-2824 Vol. 3 Núm. 8 